\title{
Cuidados de enfermagem ao paciente politraumatizado: revisão integrativa
}

\begin{abstract}
RESUMO | OBJETIVO: Descrever os cuidados de enfermagem ao paciente politraumatizado grave. MÉTODO: Revisão integrativa com buscas nas bases de dados CINAHL, MEDLINE/BIREME, IBECS, BDENF e LILACS, utilizando os MeSH (Medical Subject Headings) obtidos através da estratégia PVO e operador booleano em duas estratégias distintas: estratégia 01 "nursing care AND multiple trauma" e estratégia 02 "nursing care AND patient AND multiple trauma". RESULTADOS: Após buscas e aplicação dos critérios de elegibilidade restaram uma totalidade de 09 artigos. Nesse sentido, observou-se que os cuidados de enfermagem ao paciente politraumatizado estão ligados ao âmbito do manejo adequado com a estrutura corporal, com ênfase na técnica de imobilização; aos cuidados à restauração e manutenção da pele; à administração medicamentosa e controle da dor; e, ao monitoramento de sistemas fisiológicos. CONCLUSÃO: O cuidado de enfermagem é um instrumento complexo que abrange o ser em sua totalidade, onde na assistência ao politraumatizado várias intervenções são necessárias para que haja progressão no estado de saúde deste individuo, demonstrando a importância desta profissão em virtude das inúmeras atividades desempenhadas.
\end{abstract}

Palavras-chaves: Cuidados de enfermagem; Pacientes; Politraumatismo.

ABSTRACT | OBJECTIVE: To describe nursing care for severe polytrauma patients. METHOD: Integrative review with searches in the following databases: CINAHL, MEDLINE/BIREME, IBECS, BDENF and LILACS, using MeSH (Medical Subject Headings) obtained through the PVO strategy and Boolean operator in two different strategies: strategy 01 "nursing care AND multiple trauma "and strategy 02" nursing care AND patient AND multiple trauma". RESULTS: After searching and applying the eligibility criteria, a total of 09 articles remained. In this sense, it was observed that nursing care for polytrauma patients is linked to the scope of adequate management with body structure, with an emphasis on the immobilization technique; care for the restoration and maintenance of the skin; medication administration and pain control; and, the monitoring of physiological systems. CONCLUSION: Nursing care is a complex instrument that encompasses the being in its entirety, where in assistance to multiple trauma patients, several interventions are necessary for there to be progression in the health status of this individual, demonstrating the importance of this profession due to the numerous activities performed.

Keywords: Nursing care; Patients; Polytraum.

RESUMEN | OBJETIVO: Describir la atención de enfermería a pacientes politraumatizados graves. METODO: Revisión integrativa con búsquedas en las bases de datos CINAHL, MEDLINE/BIREME, IBECS, BDENF y LILACS, utilizando el MeSH (Medical Subject Headings) obtenido a través de la estrategia PVO y operador booleano en dos estrategias distintas: estrategia 01 "cuidado de enfermería y trauma múltiple" y estrategia 02 "cuidado de enfermería y y y trauma múltiple del paciente". RESULTADOS: Después de buscar y aplicar los criterios de elegibilidad, se mantuvieron un total de 09 artículos. En este sentido, se observó que la atención de enfermería para pacientes politraumatizados está vinculada al ámbito de un manejo adecuado con estructura corporal, con énfasis en la técnica de inmovilización; cuidado de la restauración y mantenimiento de la piel; administración de drogas y control del dolor; y, el monitoreo de los sistemas fisiológicos. CONCLUSION: La atención de enfermería es un instrumento complejo que abarca el ser en su totalidad, donde en el cuidado de la politraumatizada son necesarias varias intervenciones para que haya progresión en el estado de salud de este individuo, demostrando la importancia de esta profesión debido a las numerosas actividades realizadas.

Palavras claves: Cuidado de enfermería; Pacientes; Politrauma.

\section{Eli Carlos Martiniano}

Enfermeiro, Mestre em enfermagem pela Universidade Regional do Cariri - URCA. Especialista em Urgência e Emergência e Terapia Intensiva pelo Centro Universitário de Juazeiro do Norte - UNIJUAZEIRO.

ORCID: 0000-0002-0546-6315

\section{Anderson Marcos Vieira do Nascimento Enfermeiro, Mestrando em Saúde Pública pela Universidade Estadual da Paraíba. Membro do Grupo de Pesquisa e Estudos em Ciências da Saúde do CNPq - UEPB. ORCID:0000-0001-8575-1580}

José Rafael Eduardo Campos

Graduando em Enfermagem pelo Centro Universitário de Juazeiro do Norte UNIJUAZEIRO. Membro do Grupo de Pesquisa translacional em saúde coletiva do CNPq e bolsista do Programa Institucional de Bolsas de Iniciação Científica (PIBIC). ORCID:0000-0003-0463-4839

\section{Jessika Brenda Rafael Campos}

Enfermeira, Residente Multiprofissional com ênfase em Transplante pelo Hospital Universitário Walter Cantídio - UFC. ORCID:0000-0003-3768-5480

\begin{abstract}
Alyce Brito Barros
Graduanda em Enfermagem pelo Centro Universitário de Juazeiro do Norte UNIJUAZEIRO. Membro do Grupo de Pesquisa e Estudos em Ciências da Saúde do CNPq. ORCID:0000-0002-2775-1264

Dayse Christina Rodrigues Pereira Luz
Pós-Doutoranda em Ciências da Saúde
pela Faculdade de Medicina do ABC -
FMABC. Doutora em Ciências da Saúde
pela Faculdade de Medicina do ABC -
FMABC. Mestre em Enfermagem pela
Universidade Federal do Ceará. Docente
do Departamento de Enfermagem do
Centro Universitário de Juazeiro do Norte
- UNIJUAZEIRO.
ORCID:0000-0002-5719-3574
\end{abstract}


INTRODUÇÃO

$\Lambda$ s alterações socioeconômicas, políticas, aumento da urbanização e o desenvolvimento automobilístico fizeram emergir problemas importantes de Saúde Pública, entre eles, os acidentes e violências de trânsito. Atualmente, o Brasil ocupa o quinto lugar em números de acidentes de trânsito. Cotidianamente, 16 mil pessoas vão a óbito decorrentes de lesões traumáticas. Para cada vítima que vai a óbito muitas outras sobrevivem, entretanto, com sequelas que perduraram por toda a vida ${ }^{(1)}$.

Um dos mais importante de caráter altamente significativo em toda a área social é constituído pelo trauma. Levando em consideração que a morte por afecções cardíacas ou câncer diminui a vida, em média de 10 a 15 anos, de um cidadão, a morte que decorre de um trauma chega a alcançar valores de 30 a 40 anos de uma vida produtiva, uma vez que o problema acomete principalmente indivíduos jovens ${ }^{(2)}$.

A assistência à pessoa com traumas físicos deve ser executada de modo multiprofissional, no entanto, este é vivenciado de forma mais integralizada pelo profissional enfermeiro, que considera o cliente como ser biopsicossocial, não se detendo apenas a prática curativista, pois o cuidado de enfermagem é um processo dinâmico e complexo ${ }^{(3)}$.

Sendo o cuidado de enfermagem um instrumento baseado no julgamento e no conhecimento clínico realizado por enfermeiros, estes são atribuídos em vista à resultados reais, comportamentais, familiares ou comunitários e, percepções individuais, que são mensurados durante o processo de avaliação, para então continuidade do tratamento com implementação de ações específicas ${ }^{(4)}$.

O papel do enfermeiro na assistência ao paciente politraumatizado é fundamental e indispensável, uma vez que, a abordagem e a atenção direcionada à essas vítimas devem ser feitas de forma eficaz, indo além da integração dos sistemas de atendimento: prevenção, atendimento pré-hospitalar e hospitalar, e reabilitação. Nesse sentindo, a vítima necessita de atenção especializada e contínua não somente no atendimento inicial e na admissão, mas em todo o processo até o momento da alta hospitalar ${ }^{(1)}$.

O politraumatismo é decorrente de algum evento traumático em que ocorra grande desprendimento de energia, como acidentes de trânsito, quedas, atropelamentos, ferimentos por armas de fogo, entre outras causas que venham a causar no individuo lesões de maior gravidade. Essa problemática apresenta entre outros empasses, a oneração dos serviços públicos de saúde, além de prejuízos pessoais, econômicos e sociais. Apesar de tudo isto, esta foi negligenciada como doença por muito tempo, isso por conta de sua conotação de acidente, algo inesperado, no entanto, como qualquer outra patologia, já existe a obtenção do diagnóstico e a implementação de medidas de prevenção, assim como ações de controle a assistência ${ }^{(5)}$

Diante do exposto o estudo objetiva descrever os cuidados de enfermagem ao paciente politraumatizado grave.

\section{MÉTODO}

O estudo trata-se de uma revisão integrativa da literatura realizada a partir do modelo descrito por Mendes, Silveira e Galvão ${ }^{(6)}$, o qual se dividiu em seis etapas: a) Primeira etapa: identificação do tema e seleção da hipótese para a elaboração da revisão integrativa; b) Segunda etapa: estabelecimento de critérios para inclusão e exclusão de estudos/ amostragem e busca

\begin{tabular}{|l|c|c|}
\hline \multicolumn{2}{|l|}{ Quadro I. Estratégia PVO para obtenção de MeSHs. } \\
\hline Acrônimo & Componentes & Mesh \\
\hline Population & Pacientes & Patient \\
\hline Variables & Poli traumatismo & Multiple trauma \\
\hline Outcomes & Cuidado de enfermagem & Nursing care \\
\hline Fonte: Elaboração própria & \\
\hline
\end{tabular}


na literatura; c) Terceira etapa: definição das informações a serem extraídas dos estudos selecionados/categorização dos estudos; d) Quarta etapa: avaliação dos estudos incluídos na revisão integrativa; e) Quinta etapa: interpretação dos resultados; f) Sexta etapa: apresentação da revisão/síntese do conhecimento.

A primeira etapa foi constituída para obtenção de artigos apropriados ao objetivo do estudo, utilizou-se a estratégia de busca Population, Variables and Outcomes (PVO), descrito no quadro 01.

A segunda etapa se deu através do estabelecimento dos critérios de inclusão e exclusão a fim de nortear a busca e seleção dos artigos. Foram filtrados estudos disponíveis na íntegra e publicados entre 2013 e 2018, por apresentarem abordagens atualizadas sobre a temática. Foram incluídos: artigos originais, disponíveis nos idiomas inglês, português e espanhol; e, excluídos: artigos de revisão, reflexão, documentários, monografias, dissertações, teses, projetos, resumos e relatos; estudos repetidos em diferentes bases de dados; e, que não se enquadrassem na temática abordada, para tal, foi realizada leitura dos títulos e resumos.

Os estudos foram selecionados nas bases de dados Cumulative Index to Nursing and Allied Health Literature $(\mathrm{Cl}-$ NAHL), Medical Literature Analysis and Retrieval System Online (MEDLINE/BI-
IMAGEM 1: Fluxograma da seleção dos estudos incluídos a partir do protocolo PRISMA.

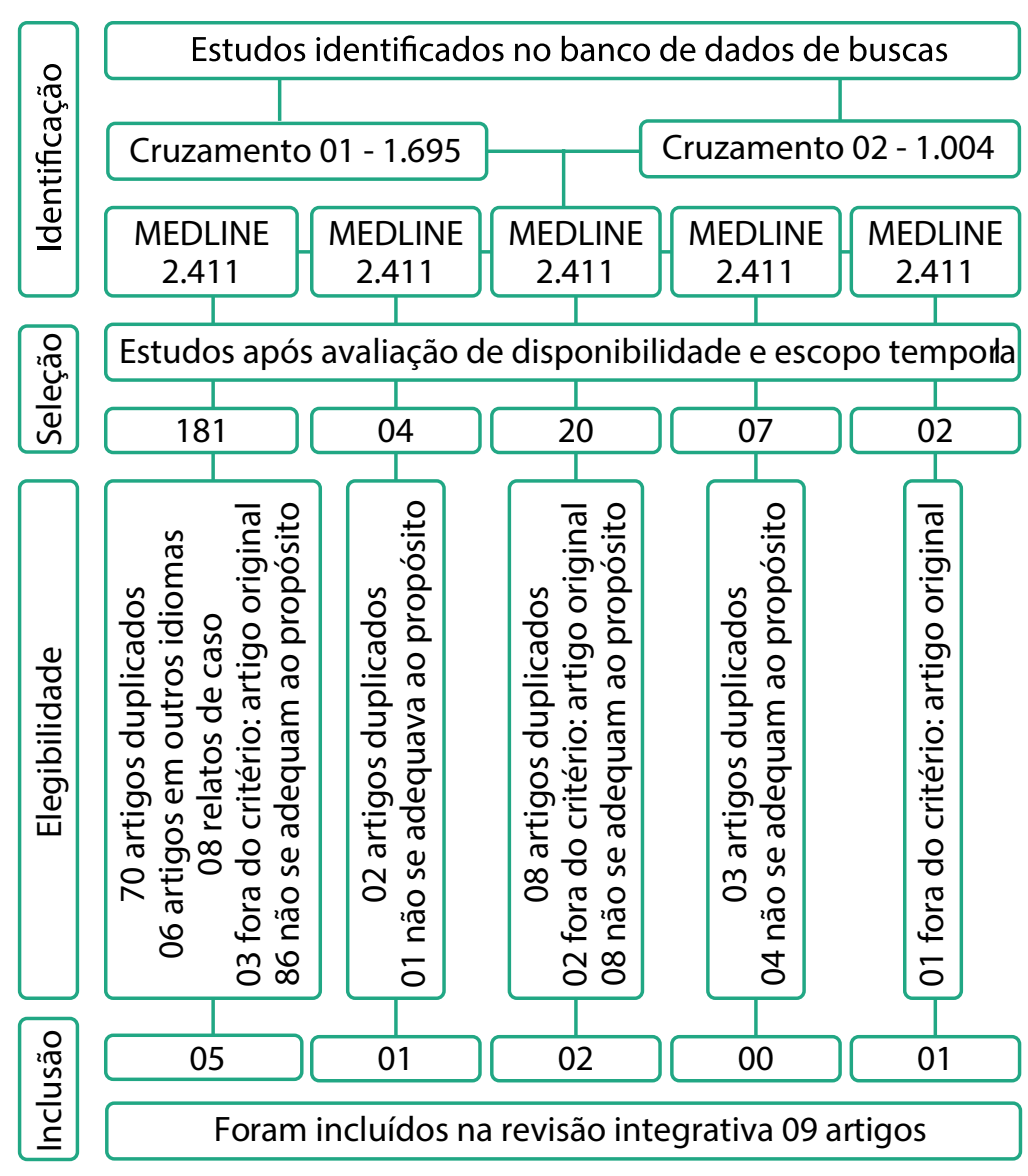

REME), Índice Bibliográfico Español em Ciencias de la Salud (IBECS), Base de Dados em Enfermagem (BDENF), Literatura Latino-americana e do Caribe em Ciências da Saúde (LILACS). Foram utilizados os Medical Subject Headings (MeSH) e operador booleano em duas estratégias distintas: estratégia 01 "nursing care AND multiple trauma" e estratégia 02 "nursing care AND patient AND multiple trauma".

A terceira etapa foi constituída da categorização dos resultados através da composição de um quadro composto por Autor/ ano/, objetivos, desfecho e número do artigo.

A quarta etapa deu- se através da classificação do nível de evidência dos artigos, proposto por Pompeo, Rossi e Galvão ${ }^{(7)}$, do nível de melhor qualidade metodológica ao nível de menor evidência que foram os seguintes:

- Nível I de evidência - Revisão sistemática ou metanálise (síntese das evidências de todos os relevantes ensaios clínicos randomizados).

- Nível II de evidência- Evidências derivadas de pelo menos um ensaio clínico randomizado, controlado, bem delimitado.

- Nível III de evidência- Evidências obtidas de ensaios clínicos bem delimitados, sem randomização.

- Nível IV de evidência- Evidências provenientes de estudos de coorte e de caso controle bem delimitados.

- Nível V de evidência- Evidência originaria de revisão sistemática de estudos descritivos e qualitativos.

A quinta etapa, ocorreu por meio das interpretações dos artigos. Nessa etapa, foi possível perceber que, nas bases pesquisadas, se houve artigos acerca da temática no período determinado.

Utilizou-se o instrumento Preferred Reporting Items for Systematic Review and Meta-Analyses (PRISMA) ${ }^{(8)}$, para demonstrar a busca e seleção dos estudos, conforme ilustrado na imagem 1: 
Quadro II. Objetivos e principais achados dos artigos.

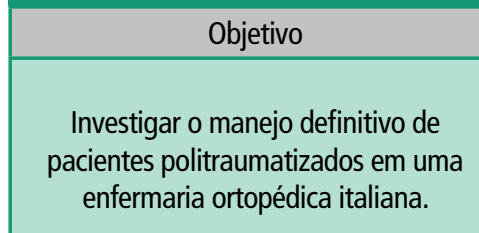

Examinar o impacto de um pacote de cuidados baseados em evidências sobre o conhecimento dos enfermeiros de emergência tailandeses sobre o manejo de pacientes com traumatismo cranioencefálico grave.

\section{Pesquisar o conhecimento dos enfermei- ros sobre o manejo da dor em pacientes com traumatismos cranioencefálicos relacionados ao combate (TBI).}

Avaliar o conhecimento de estudantes de enfermagem sobre as práticas de cuidado voltadas para pacientes politraumatizados.

Analisar o processo de implantação de um Registro de Trauma em um hospital universitário.

Fornecer recomendações baseadas nas melhores evidências disponíveis com a intenção de atuação normatizadora no cuidado adulto com traumatismo cranioencefálico grave.

Avaliar a carga de trabalho de enfermagem e verificar a correlação entre carga de trabalho e o índice de gravidade APACHE II.

Descrever as experiências de enfermeiras recém-formadas no cuidar de pessoas com amnésia pós-traumática.

Identificar os fatores relacionados à alta carga de trabalho de enfermagem necessária para vítimas de trauma internadas na UTI.
Achados

0 manejo terapêutico é realizado de acordo com a avaliação de sinais vitais, onde a dor é tratada como um sinal vital. Há enfoque no cuidado com a pele, estado nutricional e administração medicamentosa. Entre outros aspectos como educação, reabilitação (onde a mobilização difere de acordo com as lesões) e descarga (onde a alta hospitalar não significa que os cuidados parem).

Há foco na avaliação de áreas de conhecimento denominadas principais para o melhor atendimento clínico para diminuir risco de complicações e lesões cerebrais secundárias, que são: o manejo de vias aéreas e a proteção da coluna cervical, o gerenciamento de ventilação, gestão de circulação, posicionamento, reavaliação do paciente, monitorização da pressão intracraniana, gestão de dor e irritabilidade e o diagnóstico por imagem.

Este estudo identificou lacunas no conhecimento sobre o manejo da dor. Concluindo que os enfermeiros precisam de educação adicional em relação a síndromes de dor comuns, tratamentos disponíveis e um melhor entendimento do vício para proporcionar um tratamento ideal para esses pacientes.

$A$ avaliação inicial do paciente segue-se o padrão mnemônico $A B C D E$, que oferta um atendimento de acordo com a prioridade, onde de imediato deve-se realizar a abertura das vias aéreas e ofertar oxigênio, e logo em seguida realizar o controle de sangramento e reposição volêmica. A hemorragia presente nos politraumatizados é considerada como o principal fator desencadeante dos quadros de choque, e requer do socorrista atuação imediata no controle e tratamento deste sangramento.

É importante entender o conceito de que o controle de qualidade não é um meio de punir as pessoas, mas uma ferramenta para protegê-las. Nos centros de trauma mais desenvolvidos, a coleta de dados é feita profissionalmente. Existe o papel da Enfermeira de Trauma, com treinamento específico e paga exclusivamente para trabalhar em registro de trauma.

As intervenções em um atendimento inicial ao paciente traumatizado consiste no permeabilidade das vias aéreas, na ventilação adequada para prevenção de hipóxia, monitorização da circulação com foco em quadro hipotensivo e restituição de volemia, em alterações do nível de consciência ou outros déficits neurológicos, inspeção qualificada descobrindo o corpo para ter maior visibilidade das estruturas, intervenções quanto a perfusão tissular com infusão sanguínea quando necessário.

Nas atividades analisadas, encontram-se as investigações laboratoriais, medicações, medida quantitativa do débito urinário, suporte e cuidado aos familiares e pacientes que requerem dedicação exclusiva por cerca de 1 hora, tratamento para melhora da função pulmonar, cuidados com drenos, cuidados com vias aéreas artificiais e tarefas de rotina como processamento de dados, solicitação de exames e troca de informações profissionais.

Os temas que emergiram das transcrições foram: percepções de comportamento, dificuldades no manejo clínico, segurança, risco de perambulação, apoio externo, contenção e defesa da segurança do paciente.

Foram citados aspectos voltados à assistência como: o aumento do número de regiões afetadas, a gravidade fisiológica do paciente, execução de procedimentos higiênicos, trocas de curativos, monitoramento, posicionamento dos pacientes, mais encontra-se entre os fatores que impactam

\begin{tabular}{|c|c|}
\hline Artigo & Ano/Autor \\
\hline 9 & $\begin{array}{l}\text { BIZ et al., } \\
2016\end{array}$ \\
\hline 10 & $\begin{array}{l}\text { DAMKLIANG } \\
\text { et al., } 2015\end{array}$ \\
\hline 11 & $\begin{array}{l}\text { JAIMES et } \\
\text { al., } 2014\end{array}$ \\
\hline 12 & $\begin{array}{c}\text { Bezerra et } \\
\text { al., } 2015\end{array}$ \\
\hline 13 & $\begin{array}{l}\text { PARREIRA et } \\
\text { al., } 2015\end{array}$ \\
\hline 14 & $\begin{array}{l}\text { LÓPEZ-MO- } \\
\text { RALES et al., } \\
2015\end{array}$ \\
\hline 15 & $\begin{array}{l}\text { SOUZA et } \\
\text { al., } 2013\end{array}$ \\
\hline 16 & $\begin{array}{l}\text { SEARBY, } \\
2014\end{array}$ \\
\hline 17 & $\begin{array}{l}\text { NOGUEIRA } \\
\text { et al., } 2014\end{array}$ \\
\hline
\end{tabular}




\section{RESULTADOS}

Após buscas e aplicação dos critérios de elegibilidade restaram uma totalidade de 09 artigos, que foram utilizados para a construção do estudo. Entre os que não se adequavam à temática encontravam-se estudos que dissertavam com foco em outras patologias associadas, traumas psiquiátricos, avaliação de scores, protocolos e instrumentos, assistência prestada por outros que não sejam enfermeiros, ações de prevenção ao traumatismo, entre outros aspectos que não configurassem a assistência de enfermagem à pessoa com traumatismo mecânico.

Foram captados nos estudos aspectos como objetivo e principais achados, ilustrados no quadro II.

\section{DISCUSSÃO}

A atuação do profissional enfermeiro no âmbito do traumatismo, encontra-se com maior foco ao tratamento de feridas, onde está é tida como competência essencial deste profissional, que por sua vez requer conhecimentos teóricos que possam garantir a qualidade da assistência prestada, assim como também atuar na prevenção de outras deteriorações de pele relacionadas a este evento ${ }^{(1)}$.

Em 2016 um estudo investigou intervenções contidas nas evoluções de enfermagem em pacientes acompanhados por ambulatório especializado no tratamento de feridas, evidenciando que em quesitos como: aplicação de produtos, destacou-se o uso de ácidos graxos essenciais e hidrogel; os tipos de bandagem, foram relacionados à oclusão com gaze estéril e atadura de crepom em aspiral crescente; as orientações, foram quanto à realização do curativo no domicílio; a continuidade do tratamento, foi tida através de agendamento de retorno; entre outros aspectos encontrados ${ }^{(2)}$.

Outras intervenções são fundamentais na execução do processo, como: aplicar o curativo adequado à pele; manter técnica asséptica durante a realização; documentar o local, tamanho e aspecto da lesão; aplicar antibiótico tópico na área afetada; descrever vestimentas adequadas à área afetada; orientar paciente e familiares quanto aos cuidados com a ferida; e, demais ações pertinentes ${ }^{(2)}$.

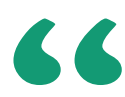

Atualmente o cuidado de enfermagem ao paciente com traumatismo raquimedular têmse tornada uma prática frequente, porém, observa-se que alguns ainda se deparam com dificuldades em aspectos como a observação do problema, manipulação, reabilitação e inserção deste individuo no ambiente familiar e social.

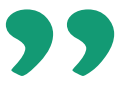

O manejo corporal adequado é primordial para que se evite o agravamento de estruturas danificadas com a lesão, esta técnica é denominada de imobilização, que consiste em um ato técnico utilizado para controlar ou impossibilitar movimentos de alguma estrutura corpórea ${ }^{(18)}$.

Atualmente o cuidado de enfermagem ao paciente com traumatismo raquimedular têm-se tornada uma prática frequente, porém, observa-se que alguns ainda se deparam com dificuldades em aspectos como a observação do problema, manipulação, reabilitação e inserção deste individuo no ambiente familiar e social. Nesse estudo foram elencados problemas vivenciados pela população com lesão raquimedular, onde encontram-se a baixo autoestima; constipação; disfunção esfincteriana; úlceras por pressão; auxílio para mobilização; auxilio espiritual; higiene corporal; preocupação financeira; alimentação; letargia e dor ${ }^{(19)}$.

A dor é um importante sinal a ser investigado no paciente com traumatismo, embora esta receba uma avaliação subjetiva, deve ser mensurada com frequência, pois este sintoma interfere diretamente no estado de saúde do indivíduo. Em estudo encontrado, enfermeiros relataram que pacientes com traumatismo craniano encefálico relataram mais intensidade de dor, porém, apenas $40 \%$ destes foram capazes de identificar corretamente a medicação apropriada para tratar a dor, constatando que estes precisam de educação adicional sobre tratamento para síndromes de dor ${ }^{(8)}$.

Além da carência profissional quanto ao aspecto dor/medicação, outro ponto que chama atenção nos estudos obtidos é classificação das diretrizes de ressuscitação cardiopulmonar, onde a ordem descrita é $\mathrm{ABCDE}$, no entanto, em Outubro de 2010 a American Heart Association publicou novas diretrizes recomendando que a ressuscitação cardiopulmonar fosse reordenada, passando então a obedecer a sequência CABDE (assistência circulatória, abertura de vias aéreas, respiração/ ventilação, avaliação neurológica, exposição e controle do ambiente) ${ }^{(20)}$. 


\section{CONCLUSÃO}

O cuidado de enfermagem é um instrumento complexo que deve abranger aspectos não somente de caráter biomédico, pois há a necessidade de contemplar o ser em todo seu estado biopsicossocial, onde o atendimento holístico e humanizado são fundamentais para o processo de recuperação do cliente com poli trauma.
Este cuidado pode ser evidenciado em vários aspectos, tendo ênfase ao desenvolvimento de ações voltadas à manutenção da pele, ao controle da mobilidade física, ao controle da dor, uma vez que o paciente politraumatizado está susceptível a elevados níveis de dor devido a sua própria condição clínica, nesse sentindo, é importantíssimo que a enfermagem esteja apta para identificar os sinais de dor que o paciente emite e intervir com o objetivo de controla-la e evitar a sua exacerbação, ao tratamento medicamentoso e à estabilização de funções fisiológicas do corpo.

Em suma, evidencia-se que a enfermagem é fundamental no processo de recuperação do paciente com politraumatismo, levando em consideração a presença constante e contínua do enfermeiro junto ao paciente e a assistência dispensada ao mesmo durante todo o processo de internação.

\section{Referências}

1. Santos MAS, Dos Santos, Oliveira GFSM, Miranda LN. Assistência de enfermagem ao paciente politraumatizado. Ciências Biológicas e de Saúde Unit. 2018 Maio; 4(2); 11-22.

2. Soares LS, De Sousa DACM, Machado ALG, Da Silva GRF. Caracterização das vítimas de traumas por acidente com motocicleta internadas em um hospital público. Uerj Nursing Journal. 2015; 1(23); DOI: https://doi. org/10.12957/reuerj.2015.15599.

3. Faria GBG, Prado TN, Lima EFA, Rogenski NMB, Borghardt AT, Massaroni L. Knowledge and practice of nurses on the care of wounds. Rev enferm UFPE on line. Recife, 2016; 10(12):4532-8. Disponivel em: <https://periodicos.ufpe.br/revistas/revistaenfermagem/article/view/11520/13408>. Acesso em: 10 de Maio de 2018.

4. De Oliveira FP, De Oliveira BGRB, Santana RF, Silva BP, Candido JSC. Classificações de intervenções e resultados de enfermagem em pacientes com feridas: mapeamento cruzado. Rev Gaúcha Enferm. 2016 jun;37(2):e55033. DOI: http://dx.doi.org/10.1590/1983- 1447.2016.02.55033

5. Mattos LS, Silvério MR. Avaliação do indivíduo vítima de politraumatismo pela equipe de enfermagem em um serviço de emergência de santa catarina. Rev. bras. promoç. saúde (Impr.). 2012; 25(2). Disponível em: <http:/l periodicos.unifor.br/RBPS/article/view/2227/2452>. Acesso em: 20 de Maio de 2018. DOI: http://dx.doi.org/10.5020/2227

6. Mendes KDS, Silveira RCC, Galvão C. Revisão integrativa: método de pesquisa para a incorporação de evidencias na saúde e na enfermagem. Texto Contexto Efer, Florianópolis; v. 17, (n.4), p. 758-64. Out-Dez, 2008.

7. Pompeo DA, Rossi LA, Galvão, CM. Revisão integrativa: etapa inicial do processo de validação de diagnóstico de enfermagem. Acta Paul Enferm; v. 22, (n.4), p. 434-8, 2009.

8. Moher D, Liberati A, Tetzlaff J, Altman DG, The PRISMA Group (2009) Preferred Reporting Items for Systematic Reviews and Meta-Analyses: The PRISMA Statement. PLoS Med 6(7): e1000097. https://doi.org/10.1371/journal. pmed.1000097

9. Biz CMD, Buffon LRN, Marin RMD, Petrova NRN. Orthopaedic nursing challenges in poly-traumatised patient management: A critical analysis of an Orthopaedic and Trauma Unit. Int J Orthop Trauma Nurs. 2016; 23: 6071. Disponível em: <https://www.orthopaedictraumanursing.com/article/ S1878-1241(16)30011-9/fulltext>. Acesso em: 23 de Abril de 2018. https:// doi.org/10.1016/j.jotn.2016.04.003

10. Damkliang J, Considine J, Kent B, Street M. Using an evidence-based care bundle to improve Thai emergency nurses' knowledge of care for patients with severe traumatic brain injury. 2015 Jul;15(4):284-92. doi: 10.1016/j. nepr.2015.03.007. Epub 2015 Mar

11. Jaimes LM, Thompson HJ, Landis CA, Warms CA. Nurses' knowledge of pain management for patients with combat-related traumatic brain injuries on rehabilitation units. Rehabil Nurs. 2015; 40(2): 74-83; quiz 81-3. Disponível em: <http://pesquisa.bvsalud.org/portal/resource/pt/mdl-24711148>. Acesso em: 26 de Abril de 2018. doi: 10.1002/rnj.156. Epub 2014 Apr 7.
12. Bezerra YCP, Medeiros RLMF de, Costa JS, Medeiros RLMF. Politraumatismo: conhecimento dos estudantes de enfermagem acerca das práticas assistenciais. Rev enferm UFPE on line., Recife, 2015; 9(11):9817-25. Disponivel em: <http://web.a-ebscohost-com.ez152.periodicos.capes.gov. $\mathrm{br} / \mathrm{ehost} / \mathrm{pdfviewer} / \mathrm{pdfviewer}$ ?vid=9\&sid=96d5be9f-a892-4691-9450-c93c23ad48ae\%40sessionmgr4009 >. Acesso em: 05 de Maio de 2018. DOI: 10.5205/reuol.8008-72925-1-ED.0911201523

13. Parreira JG, Campos T de, Perlingeiro JAG, Solda SC, Assef JC, Gonçalves $A C$ et al. Implementation of the trauma registry as a tool for quality improvement in trauma care in a brazilian hospital: the first 12 months. Rev. Col. Bras. Cir. [Internet]. 2015 Aug [cited 2018 March 15]; 42(4): 265272. Available from: http://www.scielo.br/scielo.php?script=sci_arttext\&pi$\mathrm{d}=$ S0100-69912015000500265\&lng=en. http://dx.doi.org/10.1590/010069912015004012.

14. López-Morales AB, Calderón-Dimas C, Rodríguez-Benítez G, López-Castillo $R$, García-Sandoval A. Guía de práctica clínica. Intervenciones de enfermería en la atención del adulto con traumatismo craneoencefálico grave. Rev. enferm. Inst. Mex. Seguro Soc. 2015; 23(1): 43-49, Enero. Disponível em: <http://pesquisa.bvsalud.org/portal/resource/pt/bde-29516>. Acesso em: 05 de Maio de 2018.

15. Nogueira LS, Koike KM, Sardinha DS, Padilha KG, De Sousa RMC. Carga de trabalho de enfermagem em unidades de terapia intensiva públicas e privadas. Rev Bras Ter Intensiva. 2013;25(3):225-232. DOI: 10.5935/0103507X.20130039

16. Searby A, Maude P. Graduate nurse perceptions of caring for people with posttraumatic amnesia. J Neurosci Nurs. 2014; 46(6): E16-24. Disponivel em: <http://pesquisa.bvsalud.org/portal/resource/pt/mdl-25365055>. Acesso em: 26 de Abril de 2018.

17. Nogueira LS, Domingues CA, Poggetti RS, Sousa RMC. Nursing workload in intensive care unit trauma patients: analysis of associated factors. PLoS One. 2014; 9(11): e112125. Disponível em: <http://pesquisa.bvsalud.org/ portal/resource/pt/mdl-

25375369 >. Acesso em: 28 de Abril de 2018. https://doi.org/10.1371/journal.pone. 0112125

18. Azevedo D, Soler VM. Fraturas e imobilizações em ortotraumatologia. CuidArte, Enferm. 2017; 11(2): 239-247. Disponível em: <http://pesquisa. bvsalud.org/portal/resource/pt/bde-32952>. Acesso em: 04 de Junho de 2018.

19. Silva ES, Souza SR Ferreira SMS. Sistematização da assistência de enfermagem aos clientes com traumatismo raquimedular em uma unidade de neurocirurgia. R. pesq.: cuid. fundam. online. 2011; 3(1):1542-552. Disponível em: <http://www.seer.unirio.br/index.php/cuidadofundamental/article/ view/1208/pdf_350>. Acesso em: 11 de Junho de 2018.

20. AHA - American Heart Association. Destaques da diretrizes da AHA 2010 para RCP e ACE. Editor Mary Fran Hazinski. 2010. 